\title{
Captura y selectividad del zooplancton por Utricularia foliosa (Lentibulariaceae) en la Ciénaga de Paredes, Santander, Colombia
}

\author{
Juan Sebastian Solís-Parra \& María Isabel Críales-Hernández \\ Laboratorio de Hidrobiología, Escuela de Biología, Universidad Industrial de Santander, AA 678 Bucaramanga, \\ Colombia; cbaztians@gmail.com, mcriales@uis.edu.co
}

\author{
Recibido 07-X-2015. Corregido 10-III-2016. Aceptado 08-IV-2016.
}

\begin{abstract}
Capture and selectivity of zooplankton by Utricularia foliosa (Lentibulariaceae) in the Ciénaga de Paredes, Santander, Colombia. Utricularia is a genus of carnivorous plants that capture a wide range of aquatic organisms. Most of these plants grow in environments with nutrients deficiency and have the ability to change the conditions of their microenvironment. The aim of this research was to study the selectivity in the zooplankton capture by Utricularia foliosa in the Ciénaga de Paredes. Our study was undertaken between February and November, 2014. We tried to determine if there is selection in the plant's food resources by the Czekanowski's index, and the selection degree by the Savage and Ivlev's indexes. Additionally, we studied the possible relation between the patterns of zooplankton capture and selection, with physicochemical variables in the swamp. The Czekanowski's index showed a food selection in plant resources throughout the flood pulse, with values between 0.28 and 0.41 . We also found a significant positive selection with Savage's index for Lecane sp., Alona sp., Ceriodaphnia sp., and Bosmina sp. $(\mathrm{p}<0.05)$; similar results were obtained with Ivlev's index. The intensity in the selection of each captured genus varied significantly between hydrological periods and between high and low water levels in the swamp. It was possible to identify some changes in the ammonia and nitrate concentration and some variability in the electric conductivity of the swamp, which influenced the captures made by $U$. foliosa. This is the first paper that allows an approach to understand the selection of food resources for the species, using a selectivity index, and one of the few for the genus. Rev. Biol. Trop. 64 (3): 1297-1310. Epub 2016 September 01.
\end{abstract}

Key words: Utricularia foliosa, Lentibulariaceae, carnivorous plant, selectivity index, hydrobiology, Ciénaga de Paredes.

Las cadenas tróficas, son una de las formas más efectivas de visualizar una comunidad, ya que las interacciones alimentarias, permiten rastrear el flujo de materiales y nutrientes, e identificar las relaciones más importantes de un ecosistema (Jepsen \& Winemiller, 2002). La dieta de un depredador generalmente contiene proporciones de presas diferentes a las encontradas en el ambiente de la naturaleza. Esta condición es conocida, como selección de presas (Chesson, 1983). Por ejemplo, un depredador puede exhibir una preferencia o selección positiva, cuando la abundancia de individuos de una presa en la dieta es más alta que lo encontrado en el ambiente (Begon,
Townsend, \& Harper, 2006); en caso contrario, se considera que existe selección negativa (Atienza, 1994).

Para entender las interacciones presentes dentro de las cadenas tróficas, se utilizan los análisis de selección, como aquellos empleados en estudios ecológicos de medusas, insectos acuáticos, aves, plantas carnívoras, entre otros. Estos sirven para describir la dieta del depredador y categorizar la importancia de la presa (Graham \& Kroutil, 2001; Klecka \& Boukal, 2012; Ogden, Kushlan, \& Tilmant, 2013). Con esta finalidad, se han propuesto un conjunto de índices que describen la selección y cuantifican el grado de selección; cada uno con ventajas y 
limitaciones. Los más comunes son modificaciones del más sencillo, el índice de Savage (1931), llamado también tasa de forrajeo, el cual relaciona las proporciones relativas de capturas para cada recurso, con su abundancia relativa en el ambiente. Entre los índices más utilizados, se encuentra el índice de Ivlev (1961) porque permite relacionar el grado de selección con otras variables (Pearre, 1982).

Dentro de los análisis de selección en ecosistemas acuáticos, se ha documentado la macrófita carnívora Utricularia vulgaris, que selecciona más frecuentemente a los copépodos ciclopoideos, en lugar de cladóceros del género Polyphemus, (Harms, 1999; Harms \& Johansson 2000). Sin embargo, se desconoce si esta característica es común entre todas las plantas del género Utricularia, debido a que existen pocos trabajos referentes al tema, y la mayoría son de tipo experimental (Englund \& Harms 2001; Harms, 2002; Kurbatova \& Yershov, 2009).

El género $U$. foliosa, es también una macrófita acuática, sumergida flotante-libre o mesopleustofita, de distribución cosmopolita. Crece en condiciones de bajos nutrientes (Schmidt-Mumm, 1988) y fue reportada en el Amazonas colombiano. De ella se conoce, que el porcentaje de especies capturadas, difiere del porcentaje de especies en el hábitat, lo cual confirma no aleatoriedad en la captura de las presas por parte de los utrículos. Se ha sugerido que las diferencias en la captura se deben, en parte, al comportamiento del zooplancton y macroinvertebrados cuando nadan cerca de los utrículos, en búsqueda de alimento (SanabriaAranda et al., 2006). De igual manera se ha sugerido una relación entre la inversión que realiza la planta en carnivoría, con la disponibilidad de nutrientes en el medio, particularmente, en condiciones limitantes de nitrato $\left(\mathrm{NO}_{3}{ }^{-}\right)$ (Guisande et al., 2004). Con esta información, se esperaría que el pulso de inundación o la descarga hídrica de un río, sobre las llanuras de inundación, como las que ocurren en las ciénagas, controlen la biota, y sean una de las variables responsables de las interacciones que se generan entre la planta y sus presas.
La planta $U$. foliosa fue registrada por primera vez en la Ciénaga de Paredes, Santander, Colombia en el 2013, por Críales-Hernández y Jerez-Guerrero (2016). El objetivo de esta investigación fue estudiar la selectividad en la captura de zooplancton, en la Ciénaga de Paredes, durante el pulso de inundación del año 2014. Para esto, se evaluó si existen relaciones significativas, entre la abundancia relativa de organismos del zooplancton en la ciénaga, con las presas presentes en los utrículos de la planta. Se determinó si existe selección en los recursos alimentarios, y el grado de selección durante el pulso de inundación, y se estableció si los patrones de captura están relacionados con las variables fisicoquímicas de la ciénaga.

\section{MATERIALES Y MÉTODOS}

Área de estudio: El estudio se realizó en la Ciénaga de Paredes, que pertenece al Valle Medio de la Cuenca del río Magdalena. Se encuentra localizada entre los Municipios de Sabana de Torres y Puerto Wilches, en el departamento de Santander, Colombia ( $7^{\circ} 26^{\prime}$ - $\left.7^{\circ} 29^{\prime} \mathrm{N} \& 73^{\circ} 45^{\prime}-73^{\circ} 49^{\prime} \mathrm{W}\right)$. Presenta un área aproximada de $10516.300 \mathrm{~m}^{2}$. Su afluente principal es la Quebrada La Gómez además de varios afluentes secundarios. Su principal drenaje es el Caño Peruétano (Gavilán-Díaz, 2000). La ciénaga está a una altitud de $75 \mathrm{~m}$ sobre el nivel del mar, la profundidad de la columna de agua se determina según el régimen de lluvias (Barón-Rodríguez, Gavilán-Díaz, \& Ramírez-Restrepo, 2006, Criales-Hernández \& Jerez-Guerrero, 2016). La pluviosidad de la zona presenta un ciclo bimodal definido que alcanza los $3000 \mathrm{~mm}$ anuales, con valores máximos a finales de mayo y noviembre, y mínimos de diciembre a febrero (Arias, 1985). Gavilán-Díaz, (2000) midió, durante 1998 , temperaturas máximas de $38^{\circ} \mathrm{C}$, oxígeno disuelto entre $10.8 \mathrm{mg} / \mathrm{L}$ y $0.53 \mathrm{mg} / \mathrm{L}$, tendencia a la homogeneidad en la conductividad en la columna de agua, con valores máximos de $32.0 \mu \mathrm{S} / \mathrm{cm}$ y mínimos de $8.0 \mu \mathrm{S} / \mathrm{cm}, \mathrm{y}$ valores de $\mathrm{pH}$ entre 4.57 y 8.57 . 
Fase de campo: Se realizaron cuatro muestreos, cubriendo el pulso de inundación de la Ciénaga, en el área de distribución de la planta, que corresponde a la estación Rincón de la Hoya. Los muestreos se desarrollaron dos en periodo de aguas bajas, de febrero y julio, y dos en periodos de aguas altas de mayo y noviembre 2014, teniendo en cuenta las condiciones descritas por García y Dister (1990). Durante cada muestreo, se midió la profundidad in situ, con ecosonda HONDEX ps-7, y se estimó la transparencia del agua, con un disco Secchi (Tyler, 1968). Se registró un perfil de la columna de agua de los valores de $\mathrm{pH}$, conductividad eléctrica, oxígeno disuelto y temperatura, con sonda multiparamétrica $\mathrm{HACH}$, con un grado de precisión de conductividad: $0.5 \mu \mathrm{S} / \mathrm{cm}$, temperatura: $\pm 0.3{ }^{\circ} \mathrm{C}$ y mV: $0.1 \mathrm{mV}$.

Se tomaron muestras superficiales de agua de aproximadamente $1 \mathrm{~L}$, para el análisis de nutrientes: nitrato $\left(\mathrm{NO}_{3}^{-}\right)$, amoniaco $\left(\mathrm{NH}_{4}^{+}\right)$y nitrógeno orgánico. Las muestras fueron refrigeradas $\left(4^{\circ} \mathrm{C}\right)$, almacenadas en botellas plásticas y transportadas a un laboratorio certificado para su análisis, siguiendo la metodología en APHA, AWWA y WEF (2005).

En la zona de crecimiento de la planta se tomaron muestras cuantitativas del zooplancton con Botella $\alpha$ Horizontal de $3 \mathrm{~L}$, para cada periodo hidrológico. La muestra fue tomada lo más cerca posible de las hojas de la macrófita a $50 \mathrm{~cm}$ de profundidad. Se homogenizó y se tomó una submuestra de $100 \mathrm{~mL}$; se fijó en 10 $\mathrm{mL}$ de formol buferizado al $4 \%$.

Se tomaron cinco muestras de U. foliosa (Guisande et al., 2004), por periodo hidrológico, de cada una se retiró una hoja aleatoriamente, con excepción de una planta, en el muestreo de julio, donde se extrajo una hoja adicional para determinar la eficiencia en la captura. De cada hoja se tomaron 30 utrículos con una pinza blanda y se fijaron en F.A.A (alcohol etílico de $95 \%$, ácido acético glacial, formaldehído de $37 \%$ y agua destilada) (Sandoval, 2005).

Fase de laboratorio: En el laboratorio, se extrajo cuidadosamente el contenido de los utrículos con dos agujas de disección. Se observó el contenido con un microscopio triocular Carl Zeiss Axio Lab 10, y se cuantificaron todos los organismos de zooplancton encontrados. Se identificaron con base en claves taxonómicas (Huber-Pestalozzi, Fott, Komárek, \& Förster, 1983; Gaviria, 2000; Fernando, 2002; Elías et al., 2008), hasta el nivel de género. Adicionalmente, se realizó biometría del largo de 100 utrículos, en julio, siguiendo la metodología de Guisande et al., (2000).

Para conocer la oferta potencial de géneros en el ambiente, las muestras obtenidas con Botella $\alpha$ Horizontal, de acuerdo a la turbidez de la muestra, se sedimentaron por tres días siguiendo la metodología en APHA, AWWA y WEF (2005), en probetas de $100 \mathrm{~mL}$. Se extrajo el sobrenadante, hasta alcanzar $5 \mathrm{~mL}$ de muestra y se usó una cámara de conteo Sedgwick-Rafter de $1 \mathrm{~mL}$. Se analizó el $100 \%$ de la muestra sedimentada, en cada periodo hidrológico (Gallo-Sánchez, Aguirre-Ramírez, Palacio-Baena, \& Ramírez-Restrepo, 2009).

Para determinar la dieta, se evaluó la similitud en las abundancias de los géneros capturados por periodos de aguas bajas y aguas altas mediante el uso del índice de similaridad de Morisita, modificado por Horn (1966), y se comparó ambos periodos mediante una prueba de chi-cuadrado de independencia. Después, se realizó un análisis de clúster con el mismo índice entre periodos hidrológicos.

Se evaluó la relación entre la abundancia relativa de los géneros, pertenecientes al zooplancton en el ambiente, con la abundancia relativa de las presas del mismo grupo en los utrículos de la planta. Para esto, se obtuvo la frecuencia relativa de los géneros identificados dentro de los utrículos y en las muestras sedimentadas. Con los datos obtenidos se realizó un análisis de correlación de Pearson.

La selección de los recursos alimentarios se determinó con el Índice de Similaridad Proporcional de Czekanowski (Feinsinger, Spears, $\&$ Poole, 1981): PS = 1-0,5 $\mid$ |pi-ri|, donde "ri" es la proporción de un género dentro del individuo y "pi" es la proporción de un género a su disposición en el ambiente. PS varía entre 0 (en el caso en que una población se especializa en 
el consumo de un único tipo de alimento) y 1 (cuando la población consume los recursos en igual proporción a su disponibilidad) (Galende \& Grigera, 1998).

El grado de selectividad de presas se calculó para cada género, en cada periodo hidrológico, con los índices de selectividad de Savage (1931): S = ri/pi e Ivlev (1961): Ei = (ri - pi) / (ri + pi). El índice de Savage varía desde 0 (selección negativa máxima) hasta infinito, siendo 1 el valor central de la no selección. En contraste, el índice de Ivlev varía entre - 1 (selección negativa máxima) y +1 (selección positiva máxima), siendo 0 el valor central de la no selección (Atienza, 1994). El nivel de significación del índice de Savage se evaluó según la propuesta de Manly, Mcdonald y Thomas, (1993) donde se compara el valor crítico de una prueba chi-cuadrado con un grado de libertad con el estadístico de la siguiente ecuación: ( $\mathrm{Si}$ $-1)^{2} /$ es $(\mathrm{Si})^{2}$, donde "Si" es el valor del índice de Savage para cada género. [es $(\mathrm{Si})^{2}$ ] es el error estándar del índice de Savage y se estima asumiendo a priori que no hay selección. En el índice de Ivlev no es posible evaluar el grado de significancia y por tanto se consideró un valor superior a 0.6 o inferior - 0.6 para interpretar la selectividad (Atienza, 1994).

Para establecer si la intensidad de selección de zooplancton asociada a la macrófita varía temporalmente, se evaluó el grado de significación de la variación por periodos de aguas bajas y altas mediante una prueba $U$ de Mann-Whitney y por periodos hidrológicos mediante una prueba de Kruskal-Wallis.

Por último, para establecer si los patrones de captura de zooplancton están relacionados, con las variables fisicoquímicas en la Ciénaga, se realizó un análisis de componentes principales (ACP) con los valores de $\mathrm{pH}$, conductividad eléctrica, oxígeno disuelto, temperatura, transparencia y nutrientes $\left(\mathrm{NO}_{3}^{-}, \mathrm{NH}_{4}+\mathrm{y}\right.$ nitrógeno orgánico).

Los datos se estandarizaron según el método propuesto por Guisande, Vaamonde y Barreiro (2013). Posteriormente, se seleccionaron las variables que explicaron el $90 \%$ de la varianza y a partir de estas, se realizó un análisis de correspondencia canónica (ACC) con las abundancias dentro de los utrículos de los géneros que componen la dieta de $U$. foliosa. La significancia estadística de los ejes se estimó por el test de permutación de Monte Carlo. Todos los estadísticos se calcularon con el software PAST3 (Hammer, Harper, \& Ryan, 2001).

\section{RESULTADOS}

Dieta y géneros potenciales: Durante el pulso de inundación se pudo identificar en la dieta de la macrófita un total de 12 géneros del zooplancton, que correspondieron a seis géneros de rotíferos y seis géneros de cladóceros. El género que presentó la mayor abundancia fue Lecane sp., con valores entre el $54.5 \%$ en febrero y $12.3 \%$ en mayo, seguido por Ceriodaphnia sp. y Alona sp.

El número de géneros capturados varió a través del pulso de inundación; la riqueza más alta se encontró en el mes de noviembre, correspondiente a aguas altas, y la más baja en febrero durante aguas bajas. Adicionalmente, se identificaron individuos del morfo Nauplio, solamente durante el mes de noviembre (Fig. 1).

En el punto de muestreo, se identificaron 21 géneros de zooplancton, potencialmente capturables por la macrófita, junto con el morfo Nauplio. El género con mayor abundancia fue Brachionus sp. con densidades entre 460 ind. / L (28.9\%) en el mes de noviembre y 70 ind. / L (10.0 \%) en mayo, seguido por Keratella sp. y Polyarthra sp. El cuadro 1 muestra las densidades absolutas y relativas de los géneros durante el pulso de inundación. Los porcentajes de abundancia variaron entre los periodos hidrológicos, tanto en la dieta como en los géneros potenciales (Fig. 1).

Se encontró una relación significativa entre la dieta y los periodos de aguas altas y aguas bajas (chi-cuadrado, $\mathrm{p}<0.05$ ). El índice de Morisita-Horn mostró una similaridad alta, del $84.8 \%$, entre estos dos periodos. De igual manera, ocurrió al realizar el análisis de clúster, con el índice de Morisita-Horn por periodos hidrológicos. Los meses con mayor diferencia 


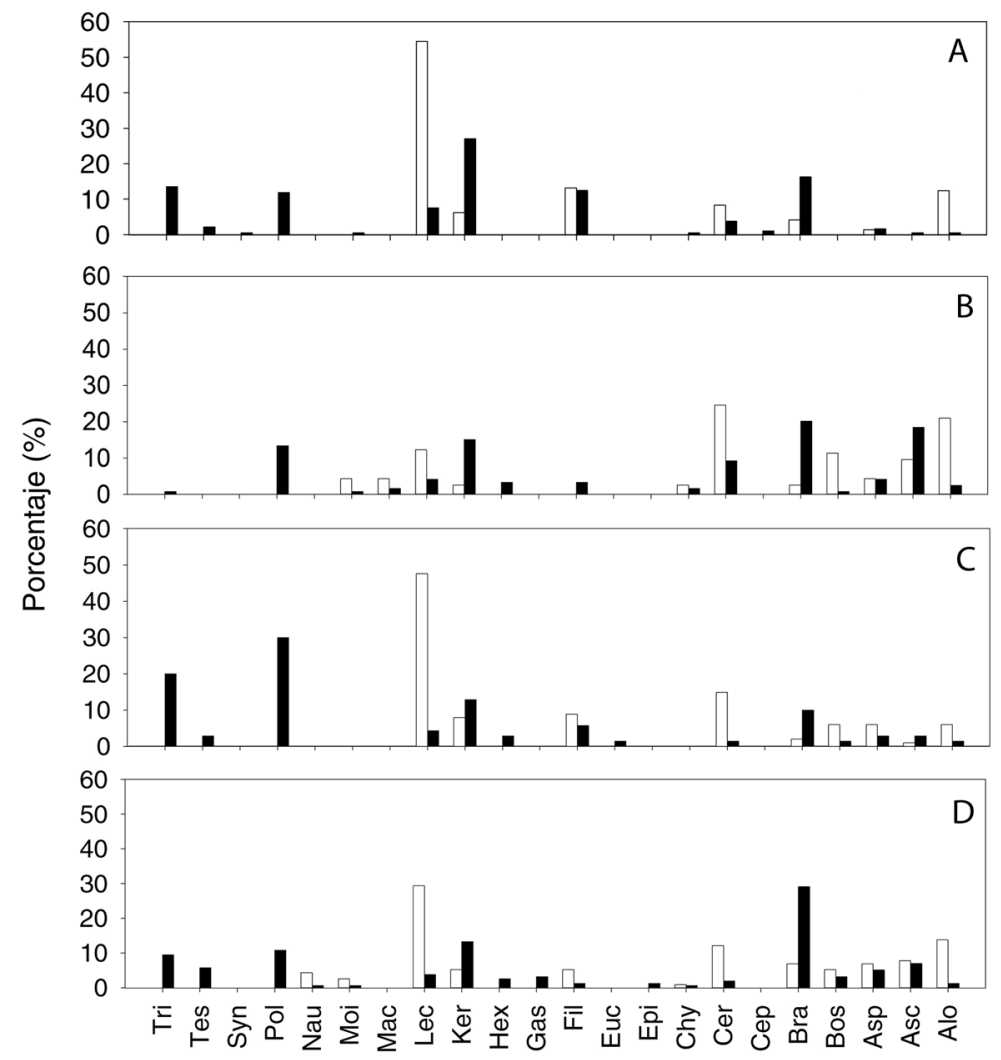

Fig. 1. Abundancia relativa de los géneros de zooplancton durante el pulso de inundación en: $\mathbf{A}=$ febrero, $\mathbf{B}=\mathrm{mayo}, \mathbf{C}=$ julio y $\mathbf{D}$ = noviembre 2014. Los géneros se representan con sus tres primeras letras (Cuadro 1). Las barras claras muestran la abundancia de los géneros capturados por $U$. foliosa y las barras oscuras, los géneros potenciales en la Ciénaga de Paredes. Fig. 1. Relative abundances of zooplankton genera throughout the flood pulse in: $\mathbf{A}=$ February, $\mathbf{B}=$ May, $\mathbf{C}=$ July and $\mathbf{D}=$ November 2014. Labels for each genus were set using their first three letters (Table. 1). Light bars represent relative abundances of genera captured by $U$. foliosa, dark bars represent relative abundances of genera in the Ciénaga de Paredes.

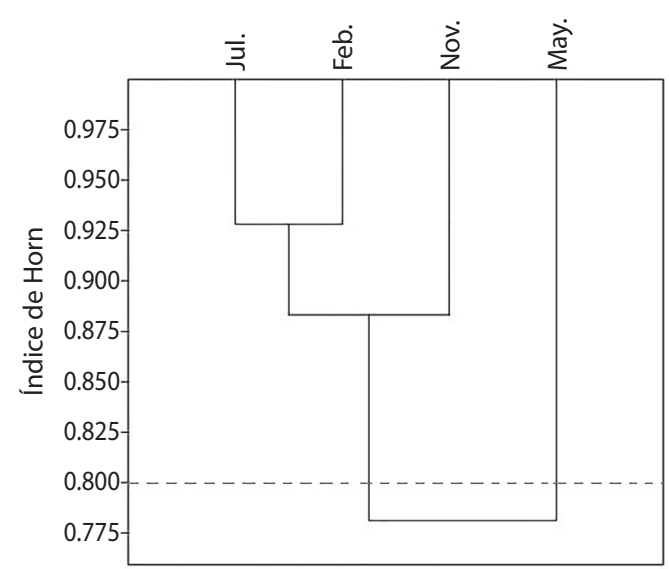

fueron julio y mayo con un valor de similaridad de $70.6 \%$ y los de mayor similitud julio y febrero con el $92.8 \%$. Se observó un agrupamiento por periodos de aguas bajas y aguas altas, según su similaridad (Fig. 2).

A través del pulso de inundación, los individuos de $U$. foliosa capturaron un número

Fig. 2. Análisis de clúster de la dieta de $U$. foliosa durante los periodos hidrológicos muestreados, febrero a noviembre 2014, Ciénaga de Paredes, Colombia.

Fig. 2. Cluster analysis using $U$. foliosa diet, during the hydrological periods sampled, February through November 2014, Ciénaga de Paredes, Colombia. 
CUADRO 1

Abundancia de zooplancton encontrada en la estación de muestreo Rincón de la Hoya,

Ciénaga de Paredes, Santander, Colombia durante el pulso de inundación del 2014

TABLE 1

Zooplankton abundance found in Rincón de la Hoya, Cienaga de Paredes, Santander, Colombia throughout the flood pulse in 2014

\begin{tabular}{|c|c|c|c|c|c|c|c|c|c|}
\hline & \multirow{2}{*}{ Código } & \multicolumn{2}{|c|}{ Febrero $\left(\mathrm{B}^{1}\right)$} & \multicolumn{2}{|c|}{ Mayo $\left(\mathrm{A}^{2}\right)$} & \multicolumn{2}{|c|}{ Julio (B) } & \multicolumn{2}{|c|}{ Noviembre (A) } \\
\hline & & $\mathrm{AB}^{3}$ (ind. $/ \mathrm{L}$ ) & $\operatorname{AR}^{4}(\%)$ & $\mathrm{AB}$ (ind./L) & AR $(\%)$ & AR (ind./L) & $\operatorname{AR}(\%)$ & $\mathrm{AB}$ (ind./L) & AR $(\%)$ \\
\hline \multicolumn{10}{|l|}{ Cladóceros } \\
\hline Alona sp. & Alo & 10.00 & 0.54 & 10.00 & 1.43 & 30.00 & 2.52 & 20.00 & 1.26 \\
\hline Bosmina sp. & Bos & - & - & 10.00 & 1.43 & 10.00 & 0.84 & 50.00 & 3.14 \\
\hline Ceriodaphnia sp. & Cer & 70.00 & 3.78 & 10.00 & 1.43 & 110.00 & 9.24 & 30.00 & 1.89 \\
\hline Chydorus sp. & Chy & 10.00 & 0.54 & - & - & 20.00 & 1.68 & 10.00 & 0.63 \\
\hline Macrothrix sp. & Mac & - & & - & - & 20.00 & 1.68 & 10.00 & 0.63 \\
\hline Moina sp. & Moi & 10.00 & 0.54 & - & - & 10.00 & 0.84 & - & - \\
\hline \multicolumn{10}{|l|}{ Rotíferos } \\
\hline Ascomorpha sp. & Asc & 10.00 & 0.54 & 20.00 & 2.86 & 220.00 & 18.49 & 110.00 & 6.92 \\
\hline Asplanchna sp. & Asp & 30.00 & 1.62 & 20.00 & 2.86 & 50.00 & 4.20 & 80.00 & 5.03 \\
\hline Brachionus sp. & $\mathrm{Bra}$ & 300.00 & 16.22 & 70.00 & 10.00 & 240.00 & 20.17 & 460.00 & 28.93 \\
\hline Cephalodella sp. & Cep & 20.00 & 1.08 & - & - & - & - & - & - \\
\hline Epiphanes sp. & Epi & - & - & - & - & - & - & 20.00 & 1.26 \\
\hline Euchlanis sp. & Euc & - & - & 10.00 & 1.43 & - & - & - & - \\
\hline Filinia sp. & Fil & 230.00 & 12.43 & 40.00 & 5.71 & 40.00 & 3.36 & 20.00 & 1.26 \\
\hline Gastropus sp. & Gas & - & - & - & - & - & - & 50.00 & 3.14 \\
\hline Hexarthra sp. & Hex & - & - & 20.00 & 2.86 & 40.00 & 3.36 & 40.00 & 2.52 \\
\hline Keratella sp. & Ker & 500.00 & 27.03 & 90.00 & 12.86 & 180.00 & 15.13 & 210.00 & 13.21 \\
\hline Lecane sp. & Lec & 140.00 & 7.57 & 30.00 & 4.29 & 50.00 & 4.20 & 60.00 & 3.77 \\
\hline Polyarthra sp. & Pol & 220.00 & 11.89 & 210.00 & 30.00 & 160.00 & 13.45 & 170.00 & 10.69 \\
\hline Synchaeta sp. & Syn & 10.00 & 0.54 & - & - & - & - & - & - \\
\hline Testudinella sp. & Tes & 40.00 & 2.16 & 20.00 & 2.86 & - & - & 90.00 & 5.66 \\
\hline Trichocerca sp. & Tri & 250.00 & 13.51 & 140.00 & 20.00 & 10.00 & 0.84 & 150.00 & 9.43 \\
\hline Nauplio & $\mathrm{Nau}$ & - & - & - & - & - & - & 10.00 & 0.63 \\
\hline
\end{tabular}

${ }^{1} \mathrm{~B}=$ periodo de aguas bajas; ${ }^{2} \mathrm{~A}=$ periodo de aguas altas; ${ }^{3} \mathrm{AB}=$ Abundancia absoluta; ${ }^{4} \mathrm{AR}=$ Abundancia relativa.

inferior de géneros a los disponibles. Solo el $33.3 \%$ fueron capturados durante febrero, 52.4 $\%$ durante mayo y noviembre; y el $42.6 \%$ en julio. Se observó que el $70.3 \%$ de los utrículos analizados contenían algún tipo de captura y el $29.7 \%$ estaban vacíos. Se debe resaltar que en el periodo hidrológico de julio, todos los utrículos presentaron algún tipo de presa en su interior y el rango del tamaño de los utrículos, durante este periodo, varió entre $400 \mu \mathrm{m}$ у $2500 \mu \mathrm{m}$.
Presencia de selección y grado de la selección: Al relacionar las abundancias relativas de los géneros identificados, dentro y fuera de los utrículos, no se encontró correlación significativa, para ninguno de los periodos hidrológicos del pulso de inundación $(\mathrm{p}>0.05)$ (Fig. 3). Se evidenció selección en los recursos alimentarios de $U$. foliosa durante todos los periodos, con valores de 0.36 en febrero, 0.41 en mayo, 0.28 en julio y 0.37 en noviembre según el índice de Czekanowski. 

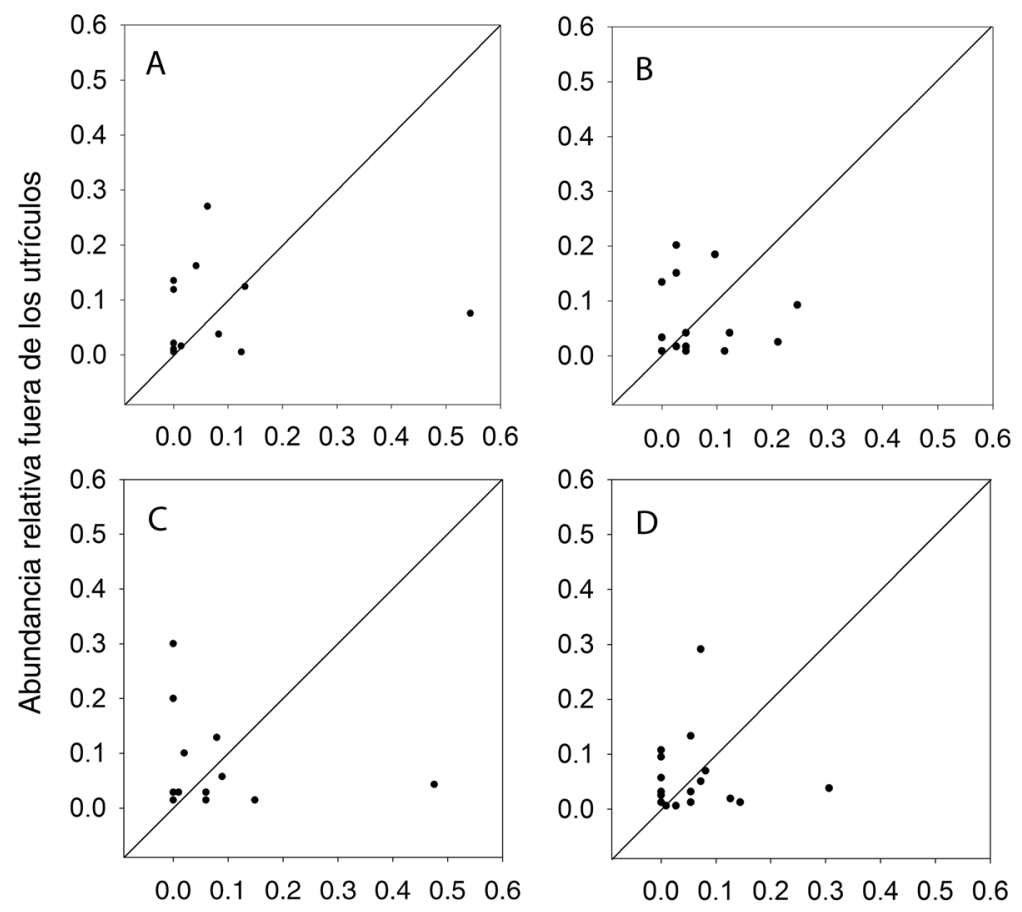

Abundancia relativa dentro de los utrículos

Fig. 3. Comparación entre la abundancia relativa de los géneros de zooplancton durante el pulso de inundación en: $\mathbf{A}=$ febrero, $\mathbf{B}=$ mayo, $\mathbf{C}=$ julio y $\mathbf{D}=$ noviembre 2014, dentro y fuera de los utrículos de la planta. La línea representa la condición en donde la abundancia dentro y fuera de los utrículos es la misma.

Fig. 3. Comparison between relative abundances of genera, throughout the flood pulse, in: $\mathbf{A}=$ February, $\mathbf{B}=$ May, $\mathbf{C}=\mathrm{July}$ and $\mathbf{D}=$ November 2014 of zooplankton inside and outside the plant bladderworts. The line represents the situation with the same abundance inside and outside the bladderworts.

Se observó selección positiva y negativa a través del pulso de inundación: Alona sp., Ascomorpha sp., Asplanchna sp., Brachionus sp., Ceriodaphnia sp., Filinia sp., Keratella sp. y Lecane sp. fueron seleccionados en todos los periodos hidrológicos. Los demás géneros fueron seleccionados al menos una vez durante el pulso.

Ocho géneros y el morfo Nauplio superaron el umbral de 0.6 (negativo o positivo) con el índice de Ivlev. El género con mayor selección positiva fue Alona sp. seguido de Bosmina sp. y Lecane sp. El género con mayor selección negativa fue Brachionus sp. seguido por Keratella sp. Se encontró significancia en la selección positiva con el índice de Savage, para Lecane sp., Alona sp., Ceriodaphnia sp., y Bosmina sp. $(\mathrm{p}<0.05)$.
Nueve géneros no fueron capturados por la macrófita en ningún periodo hidrológico, lo que da como resultado valores en el índice de Savage de 0 e Ivlev de -1 (Cuadro 2). Polyarthra sp. y Trichocerca sp. no formaron parte de la dieta, aún cuando sus abundancias fueron altas en la ciénaga (Fig. 1).

Variación de la selección: Debido a que la captura de los géneros no fue homogénea a través del pulso de inundación, solo se pudo evaluar la variación del grado de selección, para los géneros, que fueron capturados en todas las muestras de $U$. foliosa analizadas. El grado selectividad positiva sobre Alona sp., Ceriodaphnia sp. y Lecane sp. no varió significativamente entre periodos de aguas bajas y aguas altas (Mann-Whitney, $\mathrm{p}>0.05$ ). 
CUADRO 2

Índices de selección de Savage (S) e Ivlev (E) a través del pulso de inundación

TABLE 2

Savage (S) and Ivlev (E) selectivity indexes through the flood pulse

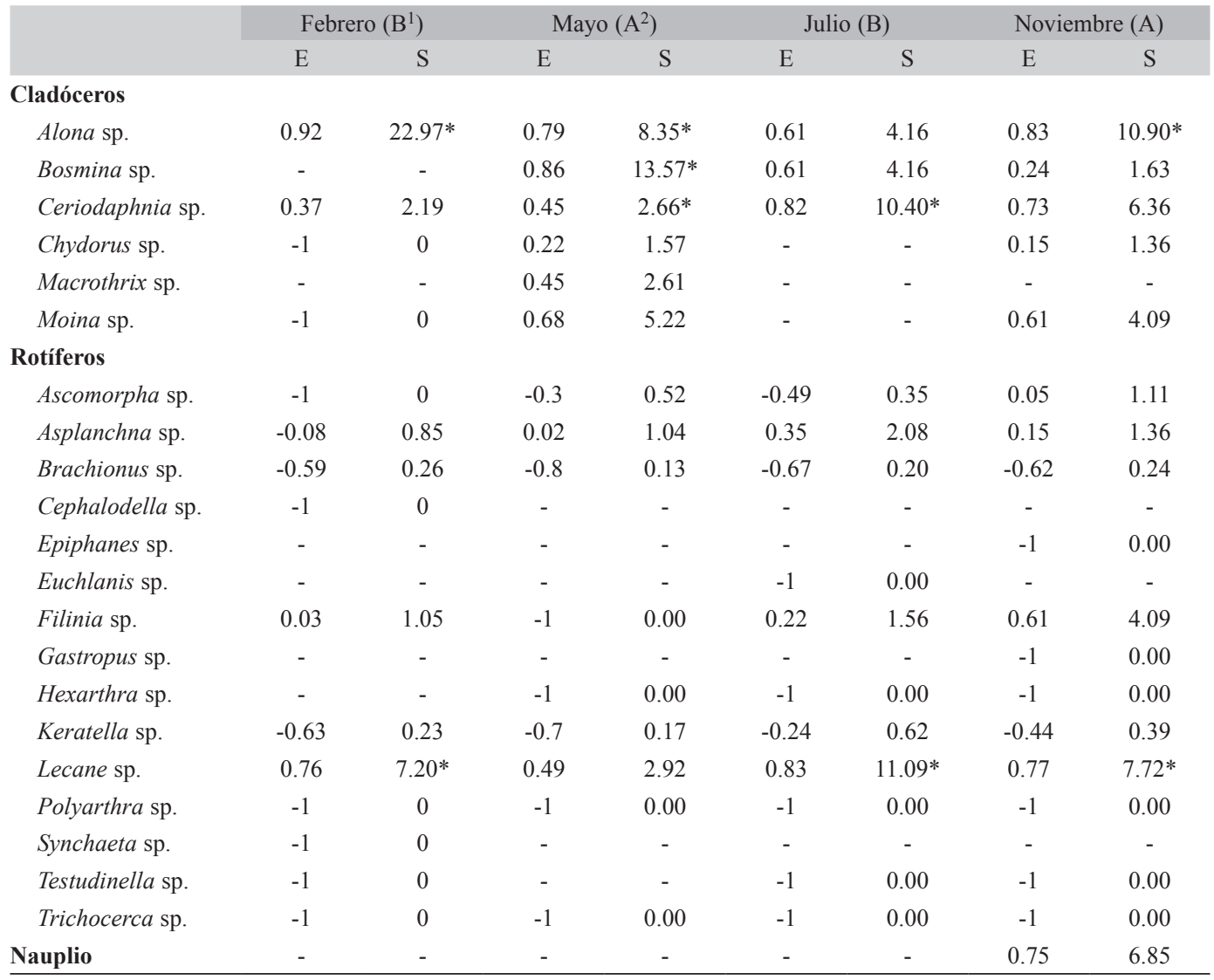

${ }^{1} \mathrm{~B}=$ periodo de aguas bajas; ${ }^{2} \mathrm{~A}=$ periodo de aguas altas. *significancia según Manly, Mcdonald y Thomas (1993) $(\mathrm{p}<0.05)$.

Sin embargo, se pudo observar, que para Alona sp. en febrero varió significativamente con respecto a los demás. Algo similar ocurrió en Ceriodaphnia sp., donde se pudo observar, que el mes de mayo varió significativamente (Kruskal-Wallis, p < 0.05), (Mann-Whitney, $\mathrm{p}<0.05)$. En Lecane sp., la selección varió significativamente (Kruskal-Wallis, $\mathrm{p}<0.05$ ) durante todo el pulso. Por último, Keratella sp. presentó una tendencia de selección negativa que no varía significativamente, entre periodos hidrológicos (Kruskal-Wallis, p>0.05) (Fig. 4).
Relación de la captura con variables fisicoquímicas: Durante el periodo de muestreo, los parámetros físicoquímicos de la ciénaga mostraron variación temporal. La temperatura promedio de la columna de agua fue de $31.3{ }^{\circ} \mathrm{C}\left(\mathrm{DE}=2.83^{\circ} \mathrm{C}\right)$, con valores máximos en febrero $\left(34.20^{\circ} \mathrm{C}\right)$ y mínimos en noviembre $\left(27.0{ }^{\circ} \mathrm{C}\right)$. La mayor conductividad eléctrica se presentó en mayo, durante el periodo de aguas bajas $(82.40 \mu \mathrm{S} / \mathrm{cm})$ y la mínima en noviembre, durante el periodo de aguas altas (9.12 $\mu \mathrm{S} / \mathrm{cm})$. El $\mathrm{pH}$ promedio del agua 

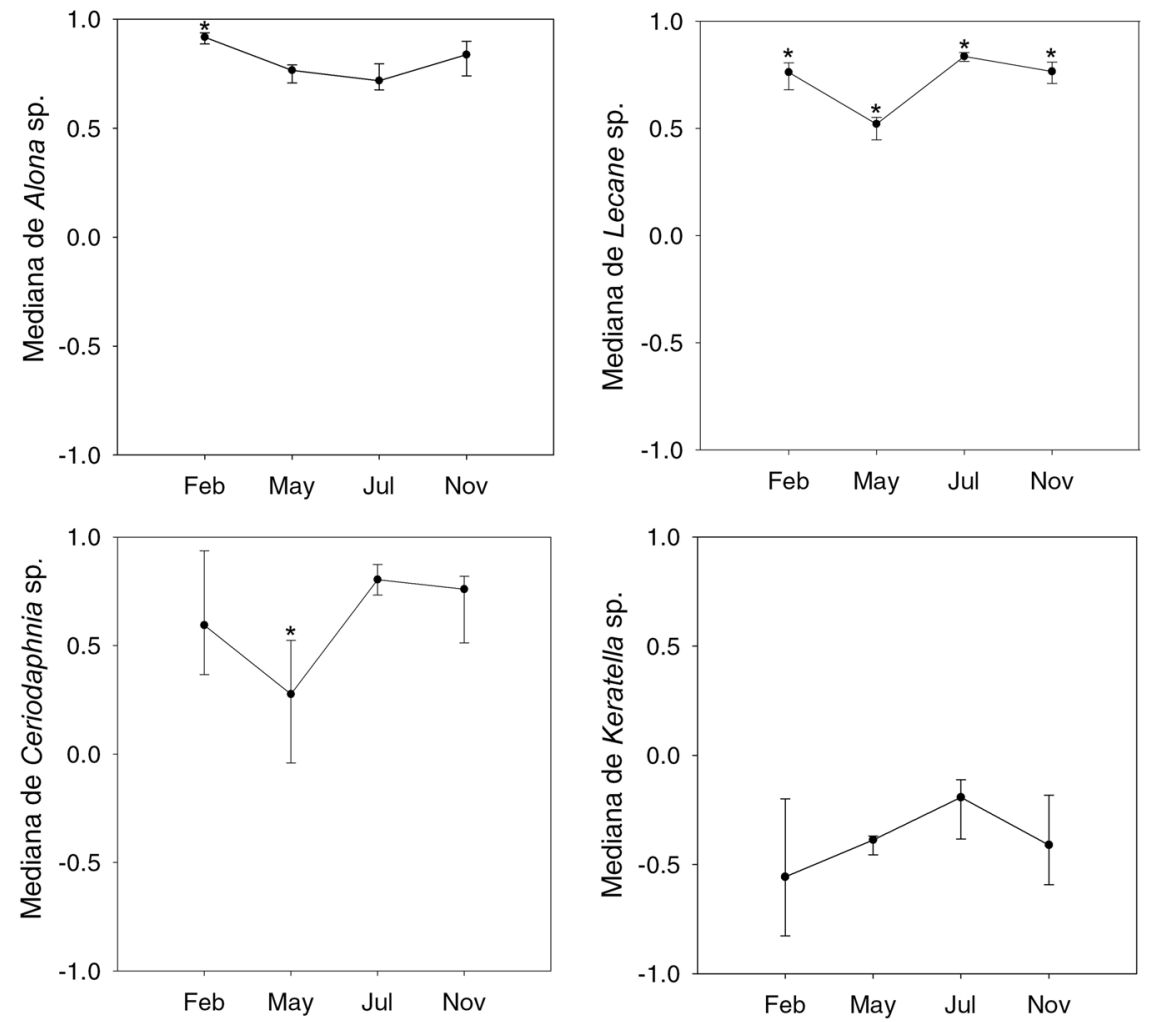

Fig. 4. Mediana del índice de Ivlev para cuatro géneros capturados por $U$. foliosa, durante el pulso de inundación, febrero a noviembre 2014, Ciénaga de Paredes, Colombia. *diferencia significativa $(\mathrm{p}<0.05)$.

Fig. 4. Ivlev Index median value for four genera captured by $U$. foliosa during the flood pulse, February through November 2014, Ciénaga de Paredes, Colombia. *significant difference $(\mathrm{p}<0.05)$.

fue $7.52(\mathrm{DE}=1.13)$, con valores máximos en febrero (8.95) y mínimos en noviembre (5.86). La mínima concentración de oxígeno disuelto se presentó en noviembre $(7.20 \mathrm{mg}$ / L) y la máxima en febrero (10.08 $\mathrm{mg} / \mathrm{L})$. La transparencia del agua varió ampliamente entre periodos de aguas bajas y aguas altas, el valor mínimo se presentó en julio $(0.32 \mathrm{~m})$ y el máximo en mayo $(1.10 \mathrm{~m})$. Por último, las concentraciones de nutrientes en el agua fueron bajas durante todo el muestreo, en promedio se encontró valores de $0.16 \mathrm{mg} / \mathrm{L}(\mathrm{DE}=0.2 \mathrm{mg}$ / L) de nitrato, $0.22 \mathrm{mg} / \mathrm{L}(\mathrm{DE}=0.07 \mathrm{mg} / \mathrm{L})$ de amoniaco y $4.07 \mathrm{mg} / \mathrm{L}(\mathrm{DE}=2.10 \mathrm{mg} / \mathrm{L})$ de nitrógeno orgánico.

El ACP aplicado a las características físicoquímicas del agua, junto con la disponibilidad de nutrientes, proporcionó un porcentaje de varianza acumulada, por los tres primeros componentes del $99.10 \%$. El primer eje correspondió al $63.09 \%$ de la varianza, el segundo al $19.62 \%$ y el tercero al $13.39 \%$. Se seleccionó la conductividad eléctrica, el nitrato, el amoniaco y el nitrógeno orgánico, por ser las variables, con mayor aporte a cada componente para realizar el ACC, con las abundancias de los géneros presentes en la dieta.

Los dos primeros ejes en el ACC fueron significativos, según la cadena de Monte Carlo $(\mathrm{p}<0.005)$. En el primer eje se encontró una correlación con la conductividad eléctrica y una correlación inversa con el nitrato y el amoniaco. En el segundo eje una fuerte correlación con el nitrógeno orgánico. La presencia de Alona sp., Asplanchna sp., Brachionus sp., Bosmina sp., Ceriodaphnia sp., Chydorus sp., 
Macrothrix sp. y Moina sp. en los utrículos, se relacionó con el aumento de la conductividad eléctrica y disminución del amoniaco y el nitrato.

Keratella sp., Lecane sp. y Filinia sp. evidenciaron una relación inversa con las mismas variables. Por último, Ascomorpha sp. mostró una relación con el nitrógeno orgánico. El ordenamiento de los individuos de $U$. folio$s a$ evidenció una separación entre los meses correspondientes a periodos de aguas bajas y periodos de aguas altas (Fig. 5).

\section{DISCUSIÓN}

Los resultados en la dieta de U. foliosa fueron similares a los de Sanabria-Aranda et al. (2006), para la misma especie, quien identificó cladóceros pertenecientes a las familias Chydoridae; Bosminidae; Daphniinae, Macrothricidae, Sydidae e Ilyocryptidae, sin embargo, estas dos últimas no se encontraron en la dieta de los individuos en la Ciénaga de Paredes. Posiblemente, esto último se debe, a que en la zona de muestreo no se encontraron individuos de estas familias, a pesar de ser reportadas previamente por Gavilán-Díaz, (2000). Adicionalmente se identificaron individuos de la familia Moinidae, que no ha sido reportada en la dieta de la planta por otros investigadores.

En cuanto al grupo Rotifera, SanabriaAranda et al. (2006), encontraron en los utrículos la presencia de las familias, Brachionidae y Lecanidae, al igual que en los resultados de este trabajo. La familia Hexarthridae, reportada en la dieta de especies del Amazonas

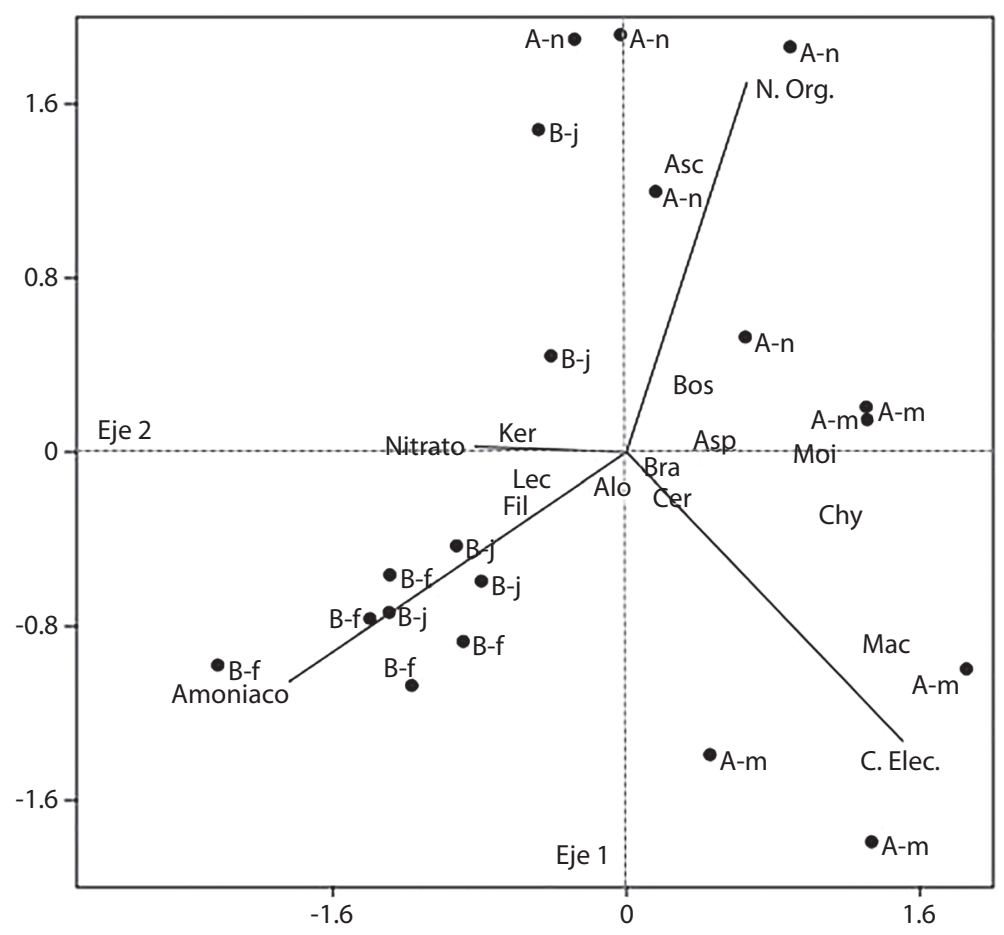

Fig. 5. Análisis de Correspondencia Canónica ACC de las especies de zooplancton capturadas por U. foliosa durante el pulso de inundación y su relación con las variables fisicoquímicas $\mathbf{C}$. Elec. $=$ conductividad eléctrica, $\mathbf{B}-\mathbf{f}=$ febrero, $\mathbf{B}-\mathbf{j}=$ julio, A-m =mayo, A-n = noviembre, los géneros en la gráfica se representan con sus tres primeras letras (Cuadro 1), Ciénaga de Paredes, Colombia.

Fig. 5. Canonical Correspondence Analysis CCA showing the zooplankton genera capture by $U$. foliosa during the flood pulse and their relationship with physic-chemical parameters. C. Elec. $=$ Electrical conductivity, B-f $=$ February, B-j = July, A-m = May, A-n = November, labels for each genus were set using their first three letters (Table 1), Ciénaga de Paredes, Colombia. 
(Sanabria-Aranda et al., 2006), no apareció en la dieta de ninguno de los individuos analizados en la ciénaga, aunque fue identificada en la estación de muestreo.

Se identificaron individuos de la familia Asplanchnidae, que no fueron reportados en la dieta de la planta por estos investigadores. Se ha sugerido que la composición de la dieta en Utricularia, depende de las condiciones químicas del agua y de la disponibilidad del recurso alimentario en ella, situación ya descrita por Mette, Wilbert y Barthlott (2000) en los géneros Utricularia australis, Utricularia vulgaris, y Utricularia macrorhiza y por Alkhalaf, Hübener y Porembski (2009).

Las variaciones en las abundancias relativas de los géneros de cladóceros potencialmente capturables, y su riqueza a través del pulso de inundación identificadas en este trabajo, son consistentes con los resultados obtenidos en la Ciénaga de Paredes por Barón-Rodríguez et al. (2006), en donde se demostraron cambios en el ensamblaje de cladóceros, causado por las fluctuaciones de la precipitación y el nivel de la columna de agua.

No existen investigaciones sobre el ensamblaje de rotíferos, en la ciénaga hasta la fecha, sin embargo, los resultados obtenidos apuntan a un patrón similar. A pesar de estas variaciones, la dieta de la macrófita fue muy similar durante todo el periodo estudiado, lo que sugiere que los cambios en las abundancias de los géneros en el ambiente; no influencian la presencia de los géneros en la dieta. La distinción en el análisis de clúster entre periodos de aguas altas y aguas bajas encontrada, puede ser considerada un indicio de la influencia de las condiciones químicas del agua, en la captura de los géneros.

Los resultados sugieren que $U$. foliosa no captura todos los géneros potenciales del zooplancton, si no que por el contrario, se limita a un grupo reducido de ellos. Algo similar a lo encontrado por Sanabria-Aranda et al. (2006). Estudios previos en la especie demuestran una relación entre el tamaño de los utrículos y la eficiencia en la captura de los géneros, es decir un aumento en la riqueza y abundancia de los grupos, con el aumento del tamaño de los utrículos (Díaz-Olarte et al., 2007).

No obstante, todas las presas potenciales del zooplancton identificadas en la ciénaga están dentro del rango de tamaño que pueden capturar los utrículos analizados, por lo tanto, el tamaño no parece ser una variable que explique la no aparición de un gran número de géneros en la dieta. Dicha situación tampoco se puede atribuir a un número bajo de capturas, puesto que el número de utrículos con capturas en la ciénaga fue alto, mayor al identificado por otros investigadores (Guisande et al., 2000).

Además, se encontró que no existieron correlaciones significativas, entre las abundancias relativas de los géneros dentro y fuera de los utrículos, similar a lo que con antelación Sanabria-Aranda et al. (2006) habían encontrado en el Amazonas colombiano. El fenómeno de la selección del zooplancton en $U$. foliosa se corroboró con el índice de Czekanowski y con los índices de Savage e Ivlev, dado que la planta seleccionó, positiva y negativamente, géneros de cladóceros y rotíferos de manera significativa. La selección de cladóceros ya ha sido identificada en individuos de las especies Utricularia vulgaris y Utricularia gibba (Harms \& Johansson, 2000; Guiral \& Rougier, 2007), sin embargo, se ha sugerido que en la captura del grupo Rotifera podría ser accidental, algo similar a lo que ocurre con el fitoplancton (Guiral \& Rougier, 2007).

Los resultados contradicen esta hipótesis y sugieren que organismos de tamaños inferiores, al de los cladóceros, tienen la capacidad de disparar el mecanismo de la trampa y por ende ser seleccionados. Se ha sugerido que las plantas del género Utricularia tienen una estrategia de predación del tipo sentarse y esperar (Harms, 1999; Harms, 2002), y por ende los géneros capturados por la macrófita exhiben diferencias, en su vulnerabilidad a la depredación, dependiendo de sus regímenes alimentarios y comportamientos (Guiral \& Rougier, 2007).

Estas diferencias pueden explicar, por qué algunos géneros no fueron capturados durante todo el pulso de inundación, aún cuando sus abundancias relativas, en la ciénaga fueron 
altas. De igual manera, se ha reconocido la presencia de glándulas dentro y fuera de los utrículos que segregan mucílagos, que atraen las presas (Wallace, 1978); el papel de estas glándulas, como una forma activa de atracción en ecosistemas acuáticos, ha sido ampliamente discutido y se debe tener en cuenta.

Adicionalmente, se ha descrito que varias especies del género Utricularia poseen estructuras en forma de antena, que se extienden desde la apertura de la trampa e imitan la forma de algas filamentosas, con las que guían y atraen géneros de cladóceros (Meyers \& Strickler, 1979), lo que explicaría el por qué se obtuvo valores altos en el índice de Ivlev y Savage, para algunos géneros de este grupo.

No se pudo identificar un patrón de captura común, para los géneros analizados con respecto a la variación del índice de Ivlev; sin embargo, fue posible establecer que el cambio en el tamaño de la columna de agua, en los periodos de aguas bajas y aguas altas, está relacionado con la captura de los géneros, aun cuando la dieta es similar durante el pulso. Las características químicas del agua parecen ser otro posible factor que explica las variaciones encontradas. Se ha encontrado que $U$. foliosa tiene la capacidad de variar su inversión en carnivoría, de acuerdo con los nutrientes que prevalecen en el agua (Guisande et al., 2004). Para estos investigadores una disminución en las concentraciones de nitrato, se ve reflejada en un mayor número de utrículos por hoja y en utrículos más grandes.

Los resultados sugieren, que no solo estas variaciones tienen una influencia sobre el tamaño y número de utrículos, sino que además, sobre los géneros que son capturados por la macrófita. Aún se desconoce exactamente el mecanismo o mecanismos con los que la planta selecciona y está por determinar, la manera en que los cambios de nutrientes modifican la interacción predador-presa. Se recomienda en futuras investigaciones profundizar en este aspecto, con el objetivo de tener una visión más amplia, sobre el fenómeno de la selección en el género.

\section{AGRADECIMIENTOS}

El trabajo se realizó en el Laboratorio de Hidrobiología de la Universidad de Santander dentro del proyecto 5715 financiado por la Vicerrectoría de Investigaciones de la Universidad Industrial de Santander. Se agradece la colaboración al Biólogo Mauricio Jerez, por su asistencia técnica en la manipulación de las muestras y a los integrantes del laboratorio de Hidrobiología de la Universidad Industrial de Santander, por su colaboración en la toma de muestras en campo y en el proceso de identificación de algunos organismos zooplanctónicos. Este documento es producto del grupo de investigación CEIAM (Centro de Estudios e Investigaciones Ambientales).

\section{RESUMEN}

Las plantas carnívoras del género Utricularia, capturan un amplio ámbito de organismos acuáticos. La mayoría se desarrollan en ambientes con carencias de nutrientes y tienen la capacidad de cambiar las condiciones de su microambiente. El objetivo de la presente investigación fue estudiar la selectividad, en la captura de zooplancton por Utricularia foliosa en la Ciénaga de Paredes; entre febrero y noviembre 2014. Se determinó si existe selección en los recursos alimentarios de la planta con el índice de Czekanowski y el grado de selección, a través de los índices de Savage e Ivlev. Se estableció la relación existente entre los patrones de captura y selección del zooplancton, con variables físicoquímicas y la disponibilidad de nutrientes en la Ciénaga. En nuestros resultados, el índice de Czekanowski evidenció selección, en los recursos alimentarios de la planta, durante todo el pulso de inundación, con valores entre 0.28 y 0.41 . Se encontró selección positiva significativa, con el índice de Savage, para los géneros Lecane sp., Alona sp., Ceriodaphnia sp., y Bosmina sp. (p $<0.05)$. Se obtuvieron resultados similares, con el índice de Ivlev. La intensidad en la selección de los géneros varió entre periodos hidrológicos y por periodos de aguas altas y bajas. Se identificó que los cambios en la concentración de amoniaco y nitrato, además de la variación en la conductividad eléctrica de la ciénaga influencian la captura que realiza $U$. foliosa. Este es el primer trabajo que permite dar un acercamiento al entendimiento de la selección de recursos alimentarios dentro de la especie a través de índices de selectividad y uno de los pocos para el género.

Palabras clave: Utricularia foliosa, Lentibulariaceae, planta carnívora, índice de selectividad, hidrobiología, Ciénaga de Paredes. 


\section{REFERENCIAS}

Alkhalaf, I. A., Hübener, T., \& Porembski, S. (2009). Prey spectra of aquatic Utricularia species (Lentibulariaceae) in northeastern Germany: The role of planktonic algae. Flora-Morphology, Distribution, Functional Ecology of Plants, 204(9), 700-708.

APHA, AWWA, \& WEF. (2005). Standard methods for the examination of water and wastewater. Washington, DC, USA: American Public Health Association (APHA).

Arias, P. (1985). Las ciénagas en Colombia. Revista Divulgación Pesquera, 22, 39-70.

Atienza, J. C. (1994). La utilización de índices en el estudio de la selección de recursos, Ardeola, 41(2), 173-175.

Barón-Rodríguez, M., R. Gavilán-Díaz, \& Ramírez-Restrepo, J. J. (2006). Variabilidad espacial y temporal en la comunidad de cladóceros de la Ciénaga de Paredes (Santander, Colombia) a lo largo de un ciclo anual. Limnética, 25, 624-635

Begon, M., Townsend, C. R., \& Harper, J. L. (2006). Ecology: from individuals to ecosystems. Malden, MA, USA: Blackwell Publishing.

Chesson, J. (1983). The estimation and analysis of preference and its relationship to foraging models. Ecology, 64(5), 1297-1304.

Críales-Hernández, M. I., \& Jerez-Guerrero, M. (2016). Primer registro de Utricularia foliosa (Lentibulariaceae) y su espectro alimentario para la Ciénaga de Paredes, Santander, Colombia. Actualidades Biológicas, 38(104), 45-51.

Díaz-Olarte, J., Valoyes-Valois, V., Guisande, C., Torres, N. N., González-Bermúdez, A., Sanabria-Aranda, L., \& Nuñez-Avellaneda, M. (2007). Periphyton and phytoplankton associated with the tropical carnivorous plant Utricularia foliosa. Aquatic Botany, 87(4), 285-291.

Englund, G., \& Harms, S. (2001). The functional response of a predatory plant preying on swarming zooplankton. Oikos, 94(1), 175-181.

Elías, M. E., Suárez-Morales, E., Gutiérrez-Aguirre, M., Silva-Briano, M., Granados Ramírez, J., \& GarfiasEspejo, T. (2008). Cladocera y Copepoda de las aguas continentales de México. Guía ilustrada. Ciudad de México, México: Universidad Nacional Autónoma de México.

Feinsinger, O., Spears, E., \& Poole, R. (1981). A simple measure of niche breadth. Ecology, 62, 27-32.

Fernando, C. H. (2002). A guide to tropical freshwater zooplankton: identification, ecology and impact on fisheries. Leiden, Holland: Backhuys.
García, L. C. \& Dister, E. (1990). Operación El Dorado: Restauración y conservación de hábitats de la planicie de inundación del medio-bajo Magdalena. Interciencia, 15(6), 396-410.

Galende, G. I. \& Grigera, D. (1998). Relaciones alimentarias de Lagidium viscacia con herbívoros introducidos en el Parque Nacional Nahuel Huapi, Argentina. Iheringia, Série Zoologia, 83, 3-10.

Gallo-Sánchez, L. J., Aguirre-Ramírez, N., Palacio-Baena, J., \& Ramírez-Restrepo, J. J. (2009). Zooplancton (Rotífera y microcrustacea) y su relación con los cambios del nivel del agua en la ciénaga de Ayapel, Córdoba, Colombia. Caldasia, 31(2), 339-353.

Gavilán-Díaz, R. A. (2000). Análisis de la diversidad en ciénagas del Magdalena Medio Santandereano (Neotrópico) con énfasis en la Comunidad zooplanctónica y el ciclo hidrológico regional (Fase I y II) (Informe Convenio UIS-Cormagdalena). Colombia.

Gaviria, S. (2000). Guía de laboratorio para identificación de Cladóceros, Copépodos y Rotíferos (Curso Sistemática del Zooplancton de las aguas continentales de Colombia). Colombia: Programa de Postgrado, Universidad de Antioquia, Medellín.

Graham, W., \& Kroutil, R. (2001). Size-based prey selectivity and dietary shifts in the jellyfish, Aurelia aurita. Journal of Plankton Research, 23(1), 67-74.

Guiral, D., \& Rougier, C. (2007). Trap size and prey selection of two coexisting bladderwort (Utricularia) species in a pristine tropical pond (French Guiana) at different trophic levels. Annales de LimnologieInternational Journal of Limnology 43(3), 147-159.

Guisande, C., Amdrade-Sessa, S., Granado-Lorencio, C., Duque, S. R., \& Núñez-Avellaneda, M. (2000). Effects on zooplankton and conductivity on tropical Utricularia foliosa investment in carnivory. Aquatic Ecology, 34(2), 137-142.

Guisande, C., Aranguren, N., Andrade-Sossa, C., Prat, N., Granado-Lorencio, C., Barrios, M. L., ... Duque, S. (2004). Relative balance of the cost and benefit associated with carnivory in the tropical Utricularia foliosa. Aquatic Botany, 80(4), 271-282.

Guisande, C., Vaamonde, A., \& Barreiro, A. (2013). Tratamiento de datos con R, Statistica y SPSS. España: Ediciones Díaz de Santos.

Hammer, Ø., Harper, D. A. T., \& Ryan, P. D. (2001). PAST: Paleontological statistics software package for education and data analysis. Palaeontologia Electronica, 4(1), 9 .

Harms, S. (1999). Prey selection in three species of the carnivorous aquatic plant Utricularia (bladderwort). Hydrobiologia, 146(4), 449-470. 
Harms, S. (2002). The effect of bladderwort (Utricularia) predation on microcrustacean prey. Freshwater Biology, 47(9), 1608-1617.

Harms, S., \& Johansson, F. (2000). The influence of prey behaviour on prey selection of the carnivorous plant Utricularia vulgaris. Hydrobiologia, 427(1), 113-120.

Horn, H. S. (1966). Measurement of "overlap" in comparative ecological studies. American naturalist, 419-424.

Huber-Pestalozzi, G., Fott, B., Komárek, J., \& Förster, K. (1983). Das Phytoplankton des süsswassers: systematik und biologie. Duitsland: E. Schweizerbart.

Ivlev, V. S. (1961). Experimental Ecology of the Feeding of Fishes. USA: Yale University Press, New Haven.

Jepsen, D. B., \& Winemiller, K. O. (2002). Structure of tropical river food webs revealed by stable isotope ratios. Oikos, 96(1), 46-55.

Klecka, J., \& Boukal, D. S. (2012). Who eats whom in a pool? A comparative study of prey selectivity by predatory aquatic insects. PloS ONE, 7(6), e37741.

Kurbatova, S. A., \& Yershov, I. Y. (2009). Crustaceans and rotifers in the predatory feeding of Utricularia. Inland Water Biology, 2(3), 271-275.

Manly, B. F. J., Mcdonald, L. L., \& Thomas, D. L. (1993) Resource Selection by Animals: Statistical Design and Analysis for Field Studies. London, United Kingdom: Chapman and Hall.

Mette, N., Wilbert, N., \& Barthlott, W. (2000). Food composition of aquatic bladderworts (Utricularia, Lentibulariaceae) in various habitats. Beitrage zur Biologie der Pflanzen, 72(1), 1-14.
Meyers, D. G., \& Strickler, J. D. (1979). Capture enhancement in a carnivorous plant: function of antennae and bristles in Utricularia vulgaris. Science, 203, 1022-1024.

Ogden, J. C., Kushlan, J. A., \& Tilmant, J. T. (2013). Prey selectivity by the wood stork. University of California Press, 78, 324-330.

Pearre Jr, S. (1982). Estimating prey preference by predators: uses of various indices, and a proposal of another based on $\chi 2$. Canadian journal of fisheries and aquatic sciences, 39(6), 914-923.

Sanabria-Aranda, L., González-Bermúdez, A., Torres, N. N., Guisande, C., Manjarrés-Hernández, A., ValoyesValois, V., \& Duque, S. R. (2006). Predation by the tropical plant Utricularia foliosa. Freshwater Biology, 51(11), 1999-2008.

Sandoval, E. (2005). Técnicas aplicadas al estudio de la anatomía vegetal (Vol. 38). Mexico: UNAM.

Savage, R. E. (1931). The relation between the feeding of the herring off the cast coast of England and the plankton of the surrounding water. Fishery Investigation, Ministry of Agriculture. Food and Fisheries, Series 2, 1-88.

Schmidt-Mumm, U. (1988). Notas sobre la vegetación acuática de Colombia, II: Fisionomía. Revista Facultad de Ciencias Universidad Javeriana, 3, 85-119.

Tyler, J. E. (1968). The secchi disc. Limnology and oceanography, 13(1), 1-6.

Wallace, R. L. (1978). Substrate selection by larvae of the sessile rotifer Ptygura beauchampi. Ecology, 59, 221-227. 